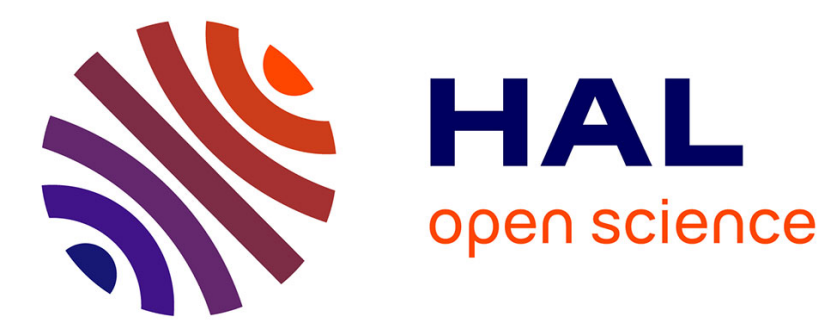

\title{
Explicit diversification benefit for dependent risks
}

Michel Dacorogna, Laila Elbahtouri, Marie Kratz

\section{To cite this version:}

Michel Dacorogna, Laila Elbahtouri, Marie Kratz. Explicit diversification benefit for dependent risks. 2015. hal-01256869

\section{HAL Id: hal-01256869 \\ https://essec.hal.science/hal-01256869}

Preprint submitted on 15 Jan 2016

HAL is a multi-disciplinary open access archive for the deposit and dissemination of scientific research documents, whether they are published or not. The documents may come from teaching and research institutions in France or abroad, or from public or private research centers.
L'archive ouverte pluridisciplinaire HAL, est destinée au dépôt et à la diffusion de documents scientifiques de niveau recherche, publiés ou non, émanant des établissements d'enseignement et de recherche français ou étrangers, des laboratoires publics ou privés. 


\title{
E \\ ESSEC \\ BUSINESS SCHOOL
}

\section{EXPLICIT DIVERSIFICATION BENEFIT FOR DEPENDENT RISKS}

\author{
RESEARCH CENTER \\ ESSEC WORKING PAPER 1522
}

2015

Michel Dacorogna

Laila Elbahtouri

Marie Kratz 


\title{
Explicit diversification benefit for dependent risks
}

\author{
Michel Dacorogna*, Laila Elbahtouri*, Marie Kratz† \\ * SCOR \\ $\dagger$ ESSEC Business School, CREAR, Paris
}

December, 2015

\begin{abstract}
We propose a new approach to analyse the effect of diversification on a portfolio of risks. By means of mixing techniques, we provide an explicit formula for the probability density function of the portfolio. These techniques allow to compute analytically risk measures as VaR or TVaR, and consequently the associated diversification benefit. The explicit formulas constitute ideal tools to analyse the properties of risk measures and diversification benefit. We use standard models, which are popular in the reinsurance industry, Archimedean survival copulas and heavy tailed marginals. We explore numerically their behavior and compare them to the aggregation of independent random variables, as well as of linearly dependent ones. Moreover, the numerical convergence of Monte Carlo simulations of various quantities is tested against the analytical result. The speed of convergence appears to depend on the fatness of the tail; the higher the tail index, the faster the convergence.
\end{abstract}

Keywords: Aggregation of risks; Archimedean copula; Clayton; Diversification (benefit); Gaussian; Gumbel; Heavy tail; Mixing technique; Pareto; Risk measure; TVaR; VaR; Weibull

\section{Introduction}

Risk and capital considerations are becoming central to the risk management of (re)insurance. In particular, the advent of Solvency 2 in the European Union is bringing along many new requirements that push the industry in this direction. For instance, Solvency 2 directive requires from (re)insurance companies to assess the capital needed to ensure their solvability. It is defined as the $99.5 \%$ quantile of the distribution of the aggregated underwriting and market risks, to cover the liabilities over one year.

Understanding the diversification benefit is essential to the business model of reinsurance companies. Diversification is at the heart of efficient risk management, capital optimization and competitive pricing. Internal models are considered as the best solution to monitor the risk within the (re)insurer portfolio and to provide the regulator with the Solvency Capital Requirements (SCR). However, regulators demand a validation of the modeling techniques used. This involves mathematical and best practice justifications of the choice of each distribution, assumption, parameter estimation, risk aggregation technique. Most models are based on Monte Carlo (MC) simulations of a large number of dependent risks. The convergence of such models is hard to prove mathematically and their stability, as a function of the number of simulations, 
is difficult to assess. The analytical approach is a way to measure the performance of the MC method, and potentially to replace it in some cases. Here, we propose this approach to improve our understanding and test the validity of model results.

Using standard models of the literature, we provide explicit expressions for the probability density function of aggregated dependent risks, allowing to compute analytically risk measures as VaR or TVaR, and consequently the diversification benefit. This can be considered as a step towards the development of methods for model validation. To achieve this, we use mixing techniques (see [10, 9]) over risk parameter values. It is a standard tool in credibility theory, but less explored for actuarial dependent risks modeling. Whereas it has been used in ruin theory (see [2]), we introduce it for risk measures and diversification benefit. Here, we concentrate on the numerical analysis in order to test the performance of the MC method and to measure the impact of the dependence (versus independence), as well as of the model choice (light versus heavy tails). Special efforts have been put in presenting in a consistent and clear way the mixing techniques approach. Following a similar path, we can extend the study to more general models for any tail index independently of the dependence parameter. It is the subject of a forthcoming paper.

The paper is organized as follows. We describe in Section 2 the framework of constructing Archimedean copulas using the mixing techniques. In Sections 3 and 4 , we present the main analytical and numerical results for two combinations of dependence structure and marginal distributions (Pareto-Clayton and Weibull-Gumbel). In Section 5, we discuss the diversification benefit as a function of the aggregation factor and the risk measures. General conclusions follow.

\section{From the mixing techniques to Archimedean copulas}

A general method for constructing multivariate Archimedean copulas has been introduced by Oakes in the bivariate case (see [10]) and extended in the multivariate case by Marshall and Olkin (see [9]). The idea behind this method is to use the mixing technique over a latent variable as a tool for dependence modeling. Introducing a latent variable to transform dependent variables into conditionally independent ones, allows to express the dependence between the variables as an Archimedean survival copula with parameter the latent variable, and to obtain the marginal distributions depending on this parameter. Namely, we have

\section{Theorem 2.1. OAKes-Marshall-OlkiN 1}

Let $\Theta$ be a positive random variable (rv) with cumulative distribution function (cdf) $F_{\Theta}$ and $X_{k}$, $k \geq 1$, be random variables (rv) such that

$$
P\left(X_{1}>x_{1}, \ldots, X_{n}>x_{n} \mid \Theta=\theta\right)=\prod_{k=1}^{n} H\left(x_{k}\right)^{\theta}
$$

$H$ being a positive function. The dependence model specified by (1) is a variant of the structure dependence generated by an Archimedean survival copula with generator $\phi=L_{\Theta}^{-1}$, where $L_{\Theta}$ denotes the Laplace transform of $F_{\Theta}$ :

$$
P\left[X_{1}>x_{1}, \ldots, X_{n}>x_{n}\right]=L_{\Theta}\left(\sum_{i=1}^{n} L_{\Theta}^{-1}\left(\bar{F}_{i}\left(x_{i}\right)\right)\right)
$$

\footnotetext{
${ }^{1}$ Note that we choose to denote this result as "the Oakes-Marshall-Olkin Theorem". It has been introduced and developed by Oakes for the bivariate case (see [10]) and generalized for any $n$ by Marshall \& Olkin (see [9]).
} 
where the marginal distributions $F_{k}$ of $X_{k}, k=1, \ldots, n$, are defined by

$$
\bar{F}_{k}(x):=1-F_{k}(x)=L_{\Theta}(-\ln H(x))
$$

By means of this mixing technique, we can provide an explicit formula for the probability density function (pdf) of aggregated risks $X_{i}, i=1, \cdots, n$, of a dependence model. Finding the appropriate choice of the function $H$ and the mixing parameter $\Theta$ to fit the marginals and the dependence model is the key step to derive via the Oakes-Marshall-Olkin theorem an explicit formula for the pdf.

With the explicit pdf $f_{S_{n}}$ of the aggregate risk $S_{n}:=\sum_{i=1}^{n} X_{i}$, denoted by $f_{n}$ when no possible confusion, we can derive the formulas for the risk measures and the diversification benefit. Recall that the diversification performance of a portfolio $S_{n}$ is measured on the gain of capital when considering a portfolio instead of a sum of standalone risks. The capital is defined by the deviation to the expectation, and the diversification benefit ([3]) at a threshold $\kappa(0<\kappa<1)$, by

$$
D_{\kappa}\left(S_{n}\right)=1-\frac{\rho_{\kappa}\left(S_{n}\right)-\mathbb{E}\left(S_{n}\right)}{\sum_{i=1}^{n}\left(\rho_{\kappa}\left(X_{i}\right)-\mathbb{E}\left(X_{i}\right)\right)}=1-\frac{\rho_{\kappa}\left(S_{n}\right)-\mathbb{E}\left(S_{n}\right)}{\sum_{i=1}^{n} \rho_{\kappa}\left(X_{i}\right)-\mathbb{E}\left(S_{n}\right)}
$$

where $\rho_{\kappa}$ denotes a risk measure at threshold $\kappa$. This indicator helps determining the optimal portfolio of the company since diversification reduces the risk and thus enhances the performance. By making sure that the diversification benefit is maximal, the company obtains the best performance for the lowest risk. However, it is important to note that $D_{\kappa}\left(S_{n}\right)$ is not a universal measure and depends on the number of risks undertaken and the chosen risk measure.

We will consider two examples of dependent models, presented in Marshall \& Olkin ([9]) (and, since, considered in various papers, as e.g. in [2]), that are useful in reinsurance context. The first model is with Pareto marginals and a Clayton structure of dependence, which is standard in reinsurance context as it captures the dependence in the tail. The second one considers Weibull marginals and a Gumbel copula; it is an interesting alternative since it combines tail dependence with thin tail distributions.

Throughout the paper, we will assume the same threshold $\kappa$ for any quantity defined w.r.t. this threshold, hence we will omit it in the notation of those quantities.

\section{Pareto marginals with Clayton survival copula}

In this example, we consider a dependent model $X=\left(X_{1}, \ldots, X_{n}\right)$ with marginals $F_{i}(i=$ $1, \ldots, n)(\alpha, \beta)$-Pareto distributed with $\alpha>1, \beta>0$, i.e. such that

$$
\overline{F_{i}}(x):=1-F_{i}(x)=\left(1+\frac{x}{\beta}\right)^{-\alpha}, \quad \forall x>0, \quad i=1, \ldots, n .
$$

If $\beta=1$, we simplify the notation writing $\alpha$-Pareto.

Recall that the quantile $q_{1}$ of order $\kappa$ of a $(\alpha, \beta)$-Pareto cdf is given by

$$
q_{1}=\operatorname{VaR}(\kappa)=\beta\left((1-\kappa)^{-1 / \alpha}-1\right)
$$

The dependence structure of the model is chosen as a Clayton survival copula with parameter $\theta>0$, defined on $[0,1]^{n}$ by

$$
C_{\theta}\left(u_{1}, \cdots, u_{n}\right)=\varphi_{\theta}^{-1}\left(\sum_{i=1}^{n} \varphi_{\theta}\left(u_{i}\right)\right)=\left(\sum_{i=1}^{n} u_{i}^{-\theta}-(n-1)\right)^{-1 / \theta}
$$


with its generator $\varphi$ given by

$$
\varphi_{\theta}(t)=t^{-\theta}-1, \quad t \in[0 ; 1]
$$

\subsection{Analytical results}

Computing directly the pdf of the aggregate risk $S_{n}$ associated to our dependent model may be a difficult task, hence the choice of using the mixing technique to work with conditional independence. We obtain the following result.

Proposition 3.1. Consider the dependent model $X=\left(X_{1}, \ldots, X_{n}\right)$ with marginals $F_{i}(i=$ $1, \ldots, n)(\alpha, \beta)$-Pareto distributed (see (5)), $\alpha>1, \beta>0$, and Clayton survival copula with parameter $\theta=1 / \alpha>0$ (see(7)). Then the $p d f f_{n}$ of the aggregate risk $S_{n}=\sum_{i=1}^{n} X_{i}$ is the one of a compound Gamma distribution (or beta distribution of the second kind with parameters $(n, \alpha)$ ) given, for $s>0$, by

$$
f_{n}(s)=\frac{\beta^{\alpha}}{B(\alpha, n)} \times \frac{s^{n-1}}{(\beta+s)^{\alpha+n}}
$$

$B$ denoting the beta function.

The choice $\theta=1 / \alpha$ is a constraint of this model. However, it can be generalized to separate the dependence from the tail index. It is the subject of a forthcoming paper.

Proof. The proof relies mainly on the application of the Oakes-Marshall-Olkin Theorem, which makes the computations of $f_{n}$ easier.

As given in [9], choosing $H(x)=e^{-x}$ for $x>0$, and the latent $\mathrm{rv} \Theta$ a Gamma $\Gamma(\alpha ; \beta)$ random variable (rv) with shape parameter $\alpha>0$ and rate parameter $\beta>0$ (or scale parameter $1 / \beta$ ) with pdf $f_{\Theta}(x)=\frac{\beta^{\alpha}}{\Gamma(\alpha)} x^{\alpha-1} e^{-\beta x} \mathbb{I}_{(x \geq 0)}$, allows to check that the model defined by (2) and (3), which corresponds to our model, satisfies (1) by the Oakes-Marshall-Olkin Theorem.

For more clarity and to be self contained, let us briefly check this statement.

The Laplace transform of $\Theta$ being $L_{\Theta}(t)=\left(1+\frac{t}{\beta}\right)^{-\alpha}$, the marginal distribution defined in (3) satisfies $L_{\Theta}(-\ln H(x))=\left(1+\frac{x}{\beta}\right)^{-\alpha}$ with this choice of $H$. It corresponds on $(0, \infty)$ to the survival cdf of a $(\alpha, \beta)$-Pareto defined in (5). Hence we get back the marginal distributions of our model.

Now, since the generator of the Archimedean survival copula defined in (2), is given by

$$
\phi(t)=L_{\Theta}(t)^{-1}=\beta\left(t^{-1 / \alpha}-1\right),
$$

we get back the structure of dependence assumed in our model (as the Archimedean generator is invariant to multiplication of the argument by a positive constant), namely (7) when taking the parameter $\theta=1 / \alpha$. 
Since conditioning $X$ by $\Theta$ transforms the dependent risks into independent conditional ones, we write the pdf $f_{n}$ of $S_{n}$ as

$$
f_{n}(s)=\int_{0}^{\infty} f_{S_{n} \mid \Theta}(s) f_{\Theta}(\theta) d \theta .
$$

With the choice of $H$ and $\Theta$, our model satisfies (1), hence the conditional rv's $X_{i} \mid \Theta=\theta$, $i=1, \cdots, n$, are i.i.d. exponentially distributed with parameter $\theta$. We deduce that their conditional sum $S_{n}=\sum_{i=1}^{n} X_{i} \mid \Theta=\theta$ is $\Gamma(n, \theta)$-distributed. So we obtain immediately that the pdf of $S_{n}$ is the one of a compound Gamma distribution and is given by

$$
f_{n}(s)=\frac{s^{n-1}}{\Gamma(n)} \int_{0}^{\infty} \theta^{n} e^{-s \theta} \frac{\beta^{\alpha}}{\Gamma(\alpha)} \theta^{\alpha-1} e^{-\beta \theta} d \theta=\frac{\Gamma(\alpha+n)}{\Gamma(\alpha) \Gamma(n)} \times \frac{\beta^{\alpha} s^{n-1}}{(\beta+s)^{\alpha+n}}
$$

hence the result (8).

Having an explicit formula (8) for the pdf $f_{n}$ of the aggregate risk $S_{n}$, we can deduce its cdf $F_{S_{n}}$ integrating $f_{n}$, and any risk measure based on $F_{S_{n}}$, as e.g. the two standard risk measures VaR and TVaR.

The Value-at-Risk of $n$ at threshold $\kappa$, denoted $q_{\kappa, n}$ or $q_{n}$, is obtained via its definition of quantile of order $\kappa$ of $F_{S_{n}}$, namely

$$
q_{n}=\operatorname{VaR}_{\kappa}\left(S_{n}\right)=F_{S_{n}}^{\leftarrow}(\kappa)
$$

$F_{S_{n}}^{\overleftarrow{ }}$ denoting the inverse of $F_{S_{n}}$. Note that using the relation between a beta distribution of second kind and a beta distribution, it may also be expressed in terms of the quantile of the beta distribution with the same parameters, $B(n, \alpha)$,

$$
q_{n}=\frac{\operatorname{VaR}_{\kappa}(B(n, \alpha))}{1-\operatorname{VaR}_{\kappa}(B(n, \alpha))} .
$$

The TVaR of $S_{n}$ at threshold $\kappa$, defined by

$$
T V a R_{n}=T \operatorname{Va} R_{\kappa}\left(S_{n}\right)=\mathbb{E}\left[S_{n} \mid S_{n} \geq q_{n}\right]
$$

( $F_{S_{n}}$ being continuous), can be computed explicitly in terms of $q_{n}$, as done in the proposition below.

For completeness, let us compute the TVaR at threshold $\kappa$ for a $(\alpha, \beta)$-Pareto cdf, assuming $\alpha>1$ (for the existence of the TVaR) and $\beta>0$. We obtain

$$
\begin{gathered}
T \operatorname{VaR}_{1}:=T \operatorname{VaR}_{\kappa}(X)=\frac{\beta^{\alpha}}{(1-\kappa)(\alpha-1)} \times \frac{\alpha q_{1}+\beta}{\left(q_{1}+\beta\right)^{\alpha}}, \text { where } q_{1} \text { is given in (6), } \\
\text { i.e. } T V \operatorname{TV} a R_{1}=\beta\left(\frac{1}{(1-1 / \alpha)(1-\kappa)^{1 / \alpha}}-1\right) .
\end{gathered}
$$

Theorem 3.1. Considering the dependent Pareto-Clayton model $X$ given in Proposition 3.1. the TVaR of the aggregate risk $S_{n}=\sum_{i=1}^{n} X_{i}$ at threshold $\kappa, 0<\kappa<1$, is given by

$$
T V a R_{n}=\frac{\beta}{(1-\kappa) B(\alpha, n)} B\left(\left(1+\frac{q_{n}}{\beta}\right)^{-1} ; \alpha-1, n+1\right)
$$


where $B(x ; a, b)$ denotes the incomplete Beta function defined by $B(x ; a, b)=\int_{0}^{x} u^{a-1}(1-u)^{b-1} d u$.

If the shape parameter $\alpha$ of the Pareto margin is such that $\alpha \in \mathbb{N} \backslash\{0,1\}$, then $T V a R_{n}$ simplifies to

$$
\begin{gathered}
T V a R_{n}=\frac{n \beta}{(1-\kappa)(\alpha-1)}\left(1-\sum_{j=0}^{\alpha-2}\left(\begin{array}{c}
n+\alpha-1 \\
j
\end{array}\right) p_{n}^{j}\left(1-p_{n}\right)^{n+\alpha-1-j}\right), \quad \text { where } p_{n}:=\left(1+\frac{q_{n}}{\beta}\right)^{-1}, \\
\text { i.e. } \quad T V a R_{n}=\frac{n \beta}{(1-\kappa)(\alpha-1)} \mathbb{P}[Y>\alpha-2],
\end{gathered}
$$

where $Y$ follows a Binomial distribution $\mathcal{B}\left(n+\alpha-1, p_{n}\right)$.

Formulas 11 and $(12)$ also hold for $n=1$, giving back 100 .

Note that another equivalent way to express the TVaR of $S_{n}$ is in terms of the hypergeometric function ${ }_{2} F_{1}$ (defined by ${ }_{2} F_{1}(a, b ; c ; z)=\sum_{k=0}^{\infty} \frac{(a)_{k}(b)_{k}}{(c)_{k}} \frac{z^{k}}{k !}$ where $\left.(a)_{k}=\frac{\Gamma(a+k)}{\Gamma(a)}\right)$, namely

$$
T \operatorname{Va} R_{n}=\frac{\beta^{\alpha}}{(\alpha-1)(1-\kappa) B(\alpha, n)} \times \frac{1}{q_{n}^{\alpha-1}} \times{ }_{2} F_{1}\left(\alpha+n, \alpha-1 ; \alpha ;-\frac{\beta}{q_{n}}\right) .
$$

Proof of Theorem 3.1

Using the definition of TVaR and Proposition 3.1, we obtain

$$
T V a R_{n}=\frac{1}{1-\kappa} \int_{q_{n}}^{\infty} s f_{n}(s) d s=\frac{\beta}{(1-\kappa) B(\alpha, n)} \int_{\frac{q_{n}}{\beta}}^{\infty} s^{n}(1+s)^{-\alpha-n} d s .
$$

The change of variables $u=(1+s)^{-1}$ in (14) gives (11). Now let us assume that $\alpha \in \mathbb{N} \backslash\{0,1\}$. We prove 12 by induction on $\alpha$.

For $\alpha=2$, computing (11) provides

$T \operatorname{VaR} R_{n}=\frac{\beta}{(1-\kappa) B(2, n)} B\left(p_{n} ; 1, n+1\right)=\frac{\beta n(n+1)}{1-\kappa} \times \frac{1-\left(1-p_{n}\right)^{n+1}}{n+1}=\frac{n \beta}{1-\kappa}\left(1-\left(1-p_{n}\right)^{n+1}\right)$,

which corresponds to 12 when replacing $\alpha$ by 2 .

Assume now that 12 is satisfied for any $2 \leq \alpha \leq k$ (and for any $n \geq 1$ ). Let us prove that it holds for $\alpha=k+1$. It comes back to prove the induction on the following expression of the incomplete beta function:

$$
B\left(p_{n} ; k, n+1\right)=\frac{(k-1) ! n !}{(n+k-1) !} B\left(p_{n} ; 1, n+k\right)-\sum_{j=1}^{k-1} \frac{(k-1) ! n !}{(n+k-j) ! j !} p_{n}^{j}\left(1-p_{n}\right)^{n+k-j} .
$$

But, since $B\left(p_{n} ; 1, n+k\right)=\frac{1}{n+k}\left(1-\left(1-p_{n}\right)^{n+k}\right)$,

$$
B\left(p_{n} ; k, n+1\right)=\frac{(k-1) ! n !}{(n+k) !}-\sum_{j=0}^{k-1} \frac{(k-1) ! n !}{(n+k-j) ! j !} p_{n}^{j}\left(1-p_{n}\right)^{n+k-j} .
$$


It is immediate to check that (15) is satisfied for $k=2$. Then, assuming that 15 holds for $k \in \mathbb{N} \backslash\{0,1\}$ (and any $n \geq 1$ ), we check that it remains true for $k+1$. An integration by part gives

$$
B\left(p_{n} ; k+1, n+1\right)=-\frac{1}{n+1} p_{n}^{k}\left(1-p_{n}\right)^{n+1}+\frac{k}{n+1} B\left(p_{n} ; k, n+2\right) .
$$

Under the inductive assumption, we can apply (15) to express $B\left(p_{n} ; k, n+2\right)$; replacing it in the previous equation provides that $B\left(p_{n} ; k+1, n+1\right)$ satisfies (15). Hence the result.

In the bivariate case $(n=2)$, the analytical expression $(13)$ can be simplified as follows.

Corollary 3.1. Consider the dependent model $X=\left(X_{1}, X_{2}\right)$ with marginals $F_{1}, F_{2}(\alpha, \beta)$ Pareto distributed, $\alpha>1, \beta>0$, and Clayton survival copula with parameter $\theta=1 / \alpha>0$. Then the TVaR of the aggregate risk $S_{2}=X_{1}+X_{2}$ at threshold $\kappa, 0<\kappa<1$, is given by

$$
T \operatorname{VaR}_{2}=\frac{\beta^{\alpha}}{(1-\kappa)(\alpha-1)} \times \frac{\alpha(1+\alpha) q_{2}^{2}+2 \beta(1+\alpha) q_{2}+2 \beta^{2}}{\left(q_{2}+\beta\right)^{1+\alpha}} .
$$

Proof. Using the definition of TVaR and Proposition 3.1 with $n=2$, gives

$$
T V a R_{2}=\frac{\alpha(1+\alpha) \beta^{\alpha}}{1-\kappa} \int_{q_{2}}^{\infty} \frac{s^{2}}{(\beta+s)^{\alpha+2}} d s
$$

from which the result follows, via the change of variables $u=s / \beta$.

Now we can deduce the main result, namely an explicit formula of the diversification benefit associated to our model, defined in (4), and denoted by $D_{n}$ when choosing TVaR as risk measure, and $D_{n}^{*}$ for VaR.

Corollary 3.2. Consider the dependent Pareto-Clayton model $X=\left(X_{i}, i \geq 1\right)$ given in Proposition 3.1. Then the diversification benefit of the aggregate risk $S_{n}=\sum_{i=1}^{n} X_{i}$ at threshold $\kappa$, $0<\kappa<1$, and associated to the risk measure $\rho$, can be expressed as:

(i) For $\rho=\operatorname{VaR}$ :

$$
D_{n}^{*}=1-\frac{\left(q_{n}-n \mathbb{E}(X)\right)}{n\left(q_{1}-\mathbb{E}(X)\right)}=1-\frac{\frac{1}{n \beta} q_{n}-\frac{1}{\alpha-1}}{(1-\kappa)^{-1 / \alpha}-\frac{\alpha}{\alpha-1}}
$$

$q_{1}$ being defined in (6) and $q_{n}$ in (9).

(ii) For $\rho=T V a R$ :

$$
D_{n}=1-\frac{\frac{(\alpha-1)}{n(1-\kappa) B(\alpha, n)} B\left(\left(1+\frac{q_{n}}{\beta}\right)^{-1} ; \alpha-1, n+1\right)-1}{\alpha\left((1-\kappa)^{-1 / \alpha}-1\right)}
$$

which simplifies, for $n=2$, to

$$
D_{2}=1-\frac{\frac{\beta^{\alpha-1}}{2(1-\kappa)} \times \frac{\alpha(1+\alpha) q_{2}^{2}+2 \beta(1+\alpha) q_{2}+2 \beta^{2}}{\left(q_{2}+\beta\right)^{1+\alpha}}-1}{\alpha\left((1-\kappa)^{-1 / \alpha}-1\right)} .
$$

Proof. (i) It is immediate knowing the fact that for a $(\alpha, \beta)$-Pareto distributed variable $\mathbb{E}(X)=\frac{\beta}{\alpha-1}$. 
(ii) Combining (10) with (11) in the definition of $D_{n}$ gives (19).

For the case $n=2,(20)$ can be directly deduced from (19). An alternative, with simpler computation, is to deduce (20) from Corollary 3.1 and the definition (4) of the diversification benefit.

\subsection{Numerical application and discussion}

The main benefit of explicit formulas is to provide exact answers for the risk measures and the diversification benefit, which are often estimated through Monte Carlo (MC) simulations, which convergence is not well known. With explicit formulas, it is thus easy to check the quality of the estimations using MC. Here we study the cases $n=2,10,100$ as illustrations and for a limited set of parameters. For the $(\alpha, \beta)$-Pareto marginals, we fix $\beta=1$.

With the results (11) (and $(12)$ when $\alpha$ is integer and $\neq 0,1$ ), we check the convergence of the simulated TVaR, varying the parameter $\alpha$ of the tail index and the aggregation factor $n$. For the parameters, we are limited here to one free, as the Clayton survival copula parameter $\theta$ relates directly to the tail index $\alpha$ with $\alpha=1 / \theta$.

First, we explore a case with very heavy tail, $\alpha=1.1$, which corresponds to tails seen for earthquake distributions, and a relatively strong dependence $(\theta \approx 0.91)$. Then we look at the case $\alpha=2$ with moderate heavy tail, followed by that of a moderate tail $\alpha=3$, which also means here a moderate dependence $\theta=1 / 3$. We run 10 sets of simulations (changing the seed of the random generator) for each of these parameters varying the number of simulations per run from 10 '000 to 10 million. We report here the average value over the 10 sets of simulations. It is worth noticing that the standard deviation of those sets decreases, as expected, with the number of simulations. For the TVaR computed for $n=2$, the standard deviation of the 10 sets varies from $32 \%$ to $4 \%$ for $\alpha=3$ and from $57^{\prime} 968 \%$ to $247 \%$ for $\alpha=1.1$ going from 100 '000 to 10 millions simulations (except for $n=100$, where we stop at 1 million due to computer limitations). It is a sign that the convergence is much slower in the case of a fatter tail. Moreover, for extremely fat tail, it does not converge even for 10 millions simulations. A similar behavior can be observed for $n=10$. Beside the gain in precision, the analytical formula can be numerically evaluated 40 times faster, resp. 580 times faster (for $\alpha=2$ and $n=10$, resp. $n=100$ for 1 million simulation) than the estimation given by Monte Carlo simulations.

We present in Figure 1 the normalized TVaR, $T V a R_{n} / n$, for being able to compare the results for the various $n$ 's. On the figure, we see that:

- The normalized TVAR of $S_{n}, T V a R_{n} / n$, decreases as $n$ increases

- The TVaR decreases as $\alpha$ increases

- The rate of convergence of $T V a R_{n} / n$ increases with $n$

- The heavier the tail, the slower the convergence

- In the case of very heavy tail and strong dependence $(\alpha=1.1$ and $\theta=0.91)$, we do not see any satisfactory convergence, even with 10 million simulations, and for any $n$

- When $\alpha=2,3$, the convergence is good from 1 million, 100'000 simulations onwards, respectively.

Looking at Table 1, we note that the convergence of the TVaR of $S_{n}$ for the Pareto parameter $\alpha=2$ is good, with a relative error going from $0.38 \%$ to $0.05 \%$ when $n$ goes from 2 to 100 , and for 


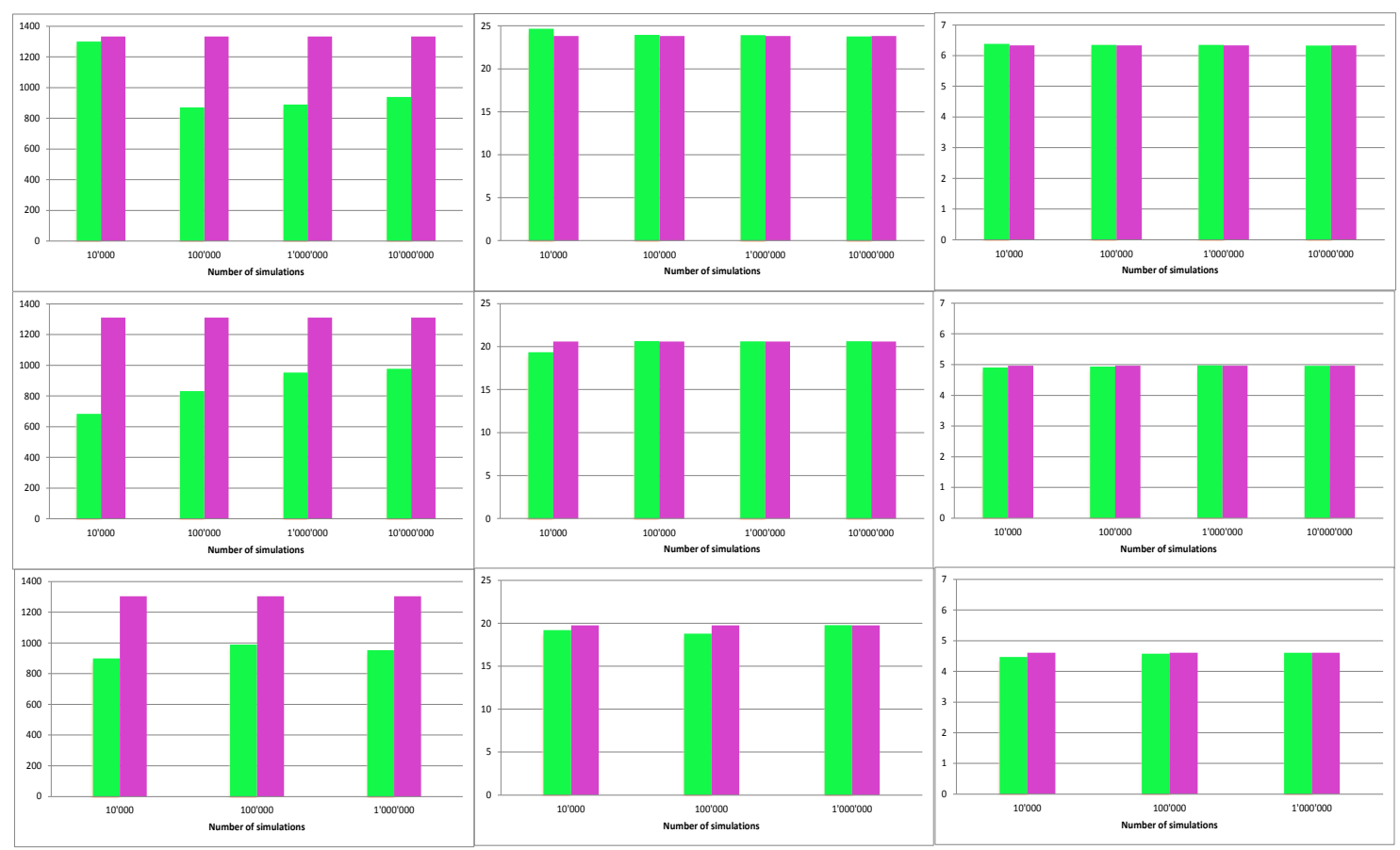

Figure 1: Convergence of the TVaR of $S_{n}$ at $99.5 \%$ for $\alpha=1.1,2,3$ from left to right, for an aggregation factor $n=2,10,100$ from up to down. The purple plots are for the analytical values and the light green ones are the average values obtained from the MC simulations. The $y$-scale gives the normalized TVaR $\left(T V a R_{n} / n\right)$ and is the same for each column.

1 million simulations. Similar relative errors are obtained for $\alpha=3$. At 10 million simulations, for $\alpha=1.1$ and $n=10$, the TVaR is still underestimated by $25 \%$, which is unacceptable for an evaluation of the solvency capital. It seems clear that MC will not give satisfactory answers with reasonable number of simulations. Thus, one might have to resort to explicit formula as derived in this paper to obtain credible values for the capital. However, increasing the number of aggregation improves the convergence. Measuring the changes with 1 million simulations, we see that from $n=2$, we have an underestimation of $50 \%$ that decreases to $27 \%$ for $n=10$ and $n=100$.

Let us turn now to the diversification benefit $D_{\kappa}\left(S_{n}\right)$ associated with $T V a R$, denoted by $D_{n}$. We use the results (20) and $(19)$ to compute it explicitly and then check the convergence of the MC. The same parameter set and the same simulations are used to produce the numbers displayed in Figure 2. The convergence of the diversification benefit follows a similar pattern as for the TVaR. Indeed, we see in Figure 2 that:

- The diversification benefit $D_{n}$ of $S_{n}$ increases with $n$

- $D_{n}$ increases with $\alpha$

- The rate of convergence of $D_{n}$ increases with $n$

- The heavier the tail, the slower the convergence

- In the case of very heavy tail and strong dependence $(\alpha=1.1$ and $\theta=0.91)$, we do not see any satisfactory convergence, even with 10 million simulations, and for any $n$

- When $\alpha=2,3$, the convergence is good from 1 million, 100'000 simulations onwards, respectively. 

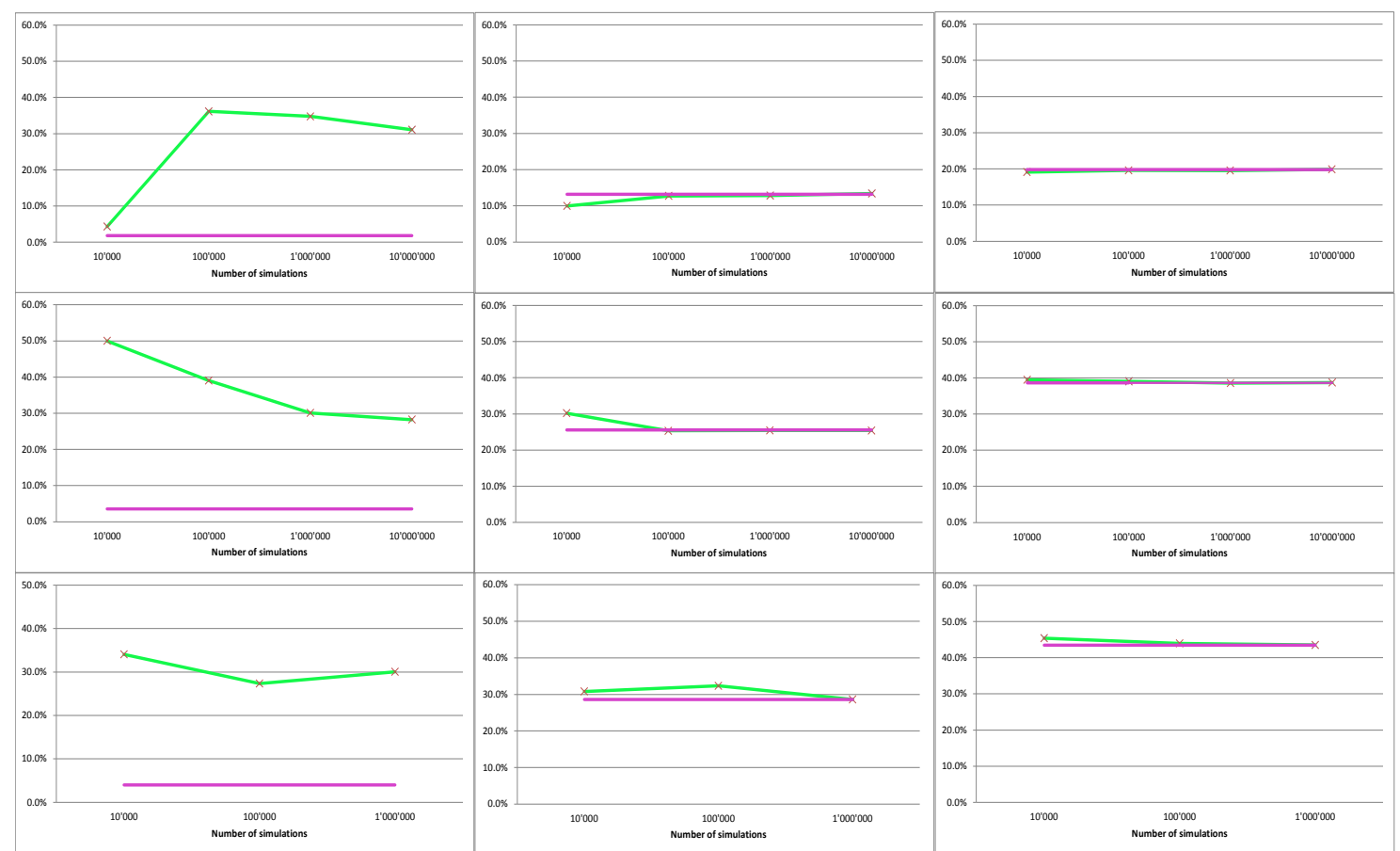

Figure 2: Convergence of the diversification benefit of $S_{n}$ (associated with TVaR at 99.5\%) for $\alpha=1.1,2,3$ (from left to right), for an aggregation factor $n=2,10,100$ (from up to down). The purple lines are for the analytical values and the light green ones are the average values obtained from the MC simulations. The $y$-scale is the same for all the graphs, for fair comparison.

For $\alpha=1.1$, the convergence is poor. In this case, we see an overestimation of the diversification benefit that is above $600 \%$ for all aggregation factors including $n=100$ (see Table 1). This big overestimation is less important since we are talking here of very small diversification benefit of the order of a few percents (3.6\% for $n=10$, for instance). We see in Figure 2 that the diversification benefit does not converge in all cases. Obviously, 10 millions simulations are not enough. The reason is that MC samples the space evenly, when we would need much more points in the tails to see a good convergence. 
Table 1: Relative errors (when comparing results obtained by MC and analytical ones) of the $T V a R_{n}$ and the diversification benefit $D_{n}$ for $S_{n}$, at $99.5 \%$ and for various $\alpha$, as a function of the aggregation factor $n$ computed with 1 million simulations.

\begin{tabular}{cccc}
\hline & $\mathrm{n}=2$ & $\mathrm{n}=10$ & $\mathrm{n}=100$ \\
\hline \hline & & & \\
$\boldsymbol{\alpha}=\mathbf{3}$ & & & \\
$T V a R_{n}$ & $0.30 \%$ & $0.14 \%$ & $-0.10 \%$ \\
$D_{n}$ & $-1.30 \%$ & $-0.25 \%$ & $0.15 \%$ \\
& & & \\
$\boldsymbol{\alpha}=\mathbf{2}$ & & & \\
$T V a R_{n}$ & $0.38 \%$ & $0.14 \%$ & $0.05 \%$ \\
$D_{n}$ & $-2.61 \%$ & $-0.44 \%$ & $-0.14 \%$ \\
& & & \\
$\boldsymbol{\alpha}=\mathbf{1 . 1}$ & & & $-26.9 \%$ \\
$T V a R_{n}$ & $-33.3 \%$ & $-27.3 \%$ & $653 \%$ \\
$D_{n}$ & $1786 \%$ & $742 \%$ & \\
\hline
\end{tabular}

In Table 1, we present the results of a convergence study as a function of the aggregation factor. The number of simulations is fixed at 1 million and we vary the aggregation factor $n$. We see a decreasing estimation error by $\mathrm{MC}$ when increasing the aggregation factor, with small errors for $\alpha=3$ and 2 and substantial errors for very fat tails and strong dependence. In the latter, we also see a systematic underestimation of the TVaR and an overestimation of the diversification benefit, whatever the aggregation factor. With the thiner tails and lower dependence, MC has a tendency to overestimate the TVaR and underestimate the diversification benefit except for $n=100$. Note that the error decrease is large between 2 and 10 but much smaller afterwards.

\section{Weibull marginals with Gumbel survival copula}

We now turn our attention to another type of distribution associated with a different copula: Weibull marginals with Gumbel dependence. Gumbel dependence is also an Archimedean survival copula presenting asymmetric dependence with strong tail dependence although less asymmetric than the Clayton survival copula. The Weibull distribution is also used in insurance particularly for survival analysis or large claim occurrences, but the tail is usually less heavy than with certain Pareto distributions. Recall that our goal is to benchmark MC methods with our analytical expressions.

Consider a dependent model $X=\left(X_{1}, \cdots, X_{n}\right)$ with marginals $F_{i}(i=1, \ldots, n),(c, \tau)$-Weibull distributed (with $c>0, \tau>0$ ), i.e. such that, for all $x \geq 0$,

$$
\overline{F_{i}}(x):=1-F_{i}(x)=e^{-c x^{\tau}} .
$$

If $c=1$, we simply speak about a $\tau$-Weibull. 
Recall that the quantile $q_{1}$ of order $\kappa(0<\kappa<1)$ of a $(c, \tau)$-Weibull cdf is given by

$$
q_{1}=\operatorname{VaR}(\kappa)=\left(-\frac{\ln (1-\kappa)}{c}\right)^{1 / \tau}
$$

The dependence structure of the model is chosen as the Gumbel suvival copula, with parameter $\theta \geq 1$, and generator $\varphi$ defined by

$$
\varphi_{\theta}(t)=(-\ln (t))^{\theta}
$$

\subsection{Analytical results}

Theorem 4.1. Consider the dependent model $X=\left(X_{1}, \ldots, X_{n}\right)$ with marginals $F_{i}(i=1, \ldots, n)$ $(c, \tau)$-Weibull-distributed with $c>0$ and $\tau=1 / 2$, and Gumbel survival copula with parameter $\theta=1 / \tau$. Then the pdf $f_{n}$ of the aggregate risk $S_{n}=\sum_{i=1}^{n} X_{i}$ at threshold $\kappa, 0<\kappa<1, n \geq 1$, is given, for $s>0$, by

$$
f_{n}(s)=\frac{c}{2 \sqrt{\pi}} \frac{\Gamma\left(n-\frac{1}{2}\right)}{\Gamma(n)} s^{-\frac{1}{2}} e^{-c \sqrt{s}}{ }_{1} F_{1}(1-n, 2-2 n ; 2 c \sqrt{s})
$$

where ${ }_{1} F_{1}(a, b ; x)$ is the Kummer confluent hypergeometric function defined on $\mathbb{R}$, with real parameters $a, b$, by ${ }_{1} F_{1}(a, b ; z)=\sum_{k=0}^{\infty} \frac{(a)_{k}}{(b)_{k}} \frac{z^{k}}{k !}$ where $(a)_{k}=\frac{\Gamma(a+k)}{\Gamma(a)}$ (see e.g. [5], p. 958). In particular, for $n=2,24$ simplifies to

$$
f_{2}(s)=\frac{c}{4}\left(\frac{1}{\sqrt{s}}+c\right) e^{-c \sqrt{s}}, \quad s>0 .
$$

The proof of this theorem is based on mixing techniques and on the following technical lemma, which helps obtaining analytical formulas.

Lemma 4.1. For $n \geq 1$, we have

$$
\partial_{s}^{n}\left(e^{-c \sqrt{s}}\right)=\frac{c(-1)^{n} \Gamma\left(n-\frac{1}{2}\right)}{2 \sqrt{\pi}} s^{\frac{1}{2}-n} e^{-c \sqrt{s}}{ }_{1} F_{1}(1-n, 2-2 n ; 2 c \sqrt{s}) .
$$

where $\partial_{s}$ denotes the partial derivative w.r.t. $s$.

Proof. We proceed by induction.

For $n=1$, Lemma 4.1 is well satisfied since $\partial_{s}\left(e^{-c \sqrt{s}}\right)=-\frac{c}{2} s^{-1 / 2} e^{-c \sqrt{s}}, \Gamma(1 / 2)=\sqrt{\pi}$, and ${ }_{1} F_{1}(0,0 ; z)=1$, for all real $z$. Suppose now that Lemma 4.1 is true for $n \geq 1$ and let us check it for $n+1$. Under the induction assumption, we have

$$
\partial_{s}^{n+1}\left(e^{-c \sqrt{s}}\right)=\frac{c(-1)^{n} \Gamma\left(n-\frac{1}{2}\right)}{2 \sqrt{\pi}} \partial_{s}\left(s^{\frac{1}{2}-n} e^{-c \sqrt{s}}{ }_{1} F_{1}(1-n, 2-2 n ; 2 c \sqrt{s})\right)
$$

Let us compute $A_{n}(s):=\partial_{s}\left(s^{\frac{1}{2}-n} e^{-c \sqrt{s}}{ }_{1} F_{1}(1-n, 2-2 n ; 2 c \sqrt{s})\right)$, using the following properties of hypergeometric functions (see e.g. [5]):

(i) $\partial_{z}\left({ }_{1} F_{1}(a, b ; z)\right)=\frac{a}{b}{ }_{1} F_{1}(a+1, b+1 ; z)$ 
(ii) ${ }_{1} F_{1}(a, 2 a ; z)=e^{z / 2}{ }_{0} F_{1}\left(a+\frac{1}{2} ; \frac{z^{2}}{16}\right)$

(iii) ${ }_{1} F_{1}(a, 2 a-1 ; z)=\frac{1}{4} e^{z / 2}\left(4{ }_{0} F_{1}\left(a-\frac{1}{2} ; \frac{z^{2}}{16}\right)+z \frac{\Gamma\left(a-\frac{1}{2}\right)}{\Gamma\left(a+\frac{1}{2}\right)}{ }_{0} F_{1}\left(a+\frac{1}{2}, \frac{z^{2}}{16}\right)\right)$

(iv) ${ }_{0} F_{1}(a ; z)=e^{-2 \sqrt{z}}{ }_{1} F_{1}\left(a-\frac{1}{2}, 2 a-1 ; 4 \sqrt{z}\right)$

where ${ }_{0} F_{1}(a ; x)$ is the confluent hypergeometric function defined by ${ }_{0} F_{1}(a ; z)=\sum_{k=0}^{\infty} \frac{1}{(a)_{k}} \frac{z^{k}}{k !}$ with $(a)_{k}=\frac{\Gamma(a+k)}{\Gamma(a)}$.

We have

$$
\begin{aligned}
A_{n}(s) & =\frac{1}{2} s^{-n} e^{-c \sqrt{s}}\left[c_{1} F_{1}(2-n, 3-2 n ; 2 c \sqrt{s})-\left(c+(2 n-1) s^{-1 / 2}\right){ }_{1} F_{1}(1-n, 2-2 n ; 2 c \sqrt{s})\right] \\
& =\frac{1}{2}(1-2 n) s^{-n-1 / 2}{ }_{0} F_{1}\left(\frac{1}{2}-n ; \frac{c^{2} s}{4}\right) \\
& =\frac{1}{2}(1-2 n) s^{-n-1 / 2} e^{-c \sqrt{s}}{ }_{1} F_{1}(-n,-2 n ; 2 c \sqrt{s})
\end{aligned}
$$

using (i) in the first equality, then both (ii) and (iii) in the second one, and (iv) in the last one. We can conclude that Lemma 4.1 is satisfied for $n+1$.

For the proof of Theorem 4.1, we will also need the following relation for $n \geq 0$ and $a, b \in \mathbb{R}$ (see e.g. [1]), we have

$$
\begin{aligned}
I_{n, b}(a)= & \int_{0}^{\infty} x^{n-1 / 2} e^{-a x-b / x} d x=(-1)^{n} \partial_{a}^{n}\left(I_{0, b}(a)\right) \\
& \text { with } I_{0, b}(a)=\int_{0}^{\infty} x^{-1 / 2} e^{-a x-\frac{b}{x}} d x=\sqrt{\frac{\pi}{a}} e^{-2 \sqrt{a b}}
\end{aligned}
$$

when differentiating $I_{n, b}(a)$ with respect to the parameter $a$.

Proof of Theorem 4.1. As given in [9], it is enough to choose $H(x)=e^{-x}(x>0)$ and $\Theta$ a Lévy-distributed $\left(0, c^{2} / 2\right)$ positive $\mathrm{rv}$ with $c>0$, and pdf $f_{\Theta}(x)=\frac{c}{2 \sqrt{\pi x^{3}}} e^{-\frac{c 2}{4 x}}$, for $x \geq 0$, then to apply the Oakes-Marshall-Olkin theorem.

Computing the Laplace transform of $\Theta$ via the change of variables $u=\theta^{-1}$, gives

$$
L_{\Theta}(t)=\int_{0}^{\infty} e^{-\theta t} f_{\Theta}(\theta) d \theta=I_{0, t}\left(c^{2} / 4\right)=e^{-c \sqrt{t}} .
$$

So, with this choice of $H$, the marginal distribution defined in (3) satisfies $L_{\Theta}(-\ln H(x))=e^{-c \sqrt{x}}$. It corresponds on $(0, \infty)$ to the survival cdf of a Weibull $(c, 1 / 2)$ defined in (21). Hence we get back the marginal distributions of our model.

Now, since the generator of the Archimedean survival copula defined in (2), is given by

$$
\phi(t)=L_{\Theta}(t)^{-1}=\frac{1}{c^{2}}(-\ln (t))^{2}
$$

we get back the structure of dependence assumed in our model, namely (23) when taking the parameter $\theta=2$. 
For the 2nd part of the proof, we can write, as in the proof of Proposition 3.1 .

$$
f_{n}(s)=\int_{0}^{\infty} f_{S_{n} \mid \Theta}(s) f_{\Theta}(\theta) d \theta=\frac{c s^{n-1}}{2 \sqrt{\pi} \Gamma(n)} \int_{0}^{\infty} \theta^{(n-1)-1 / 2} e^{-s \theta-\frac{c^{2}}{4 \theta}} d \theta .
$$

Therefore, using 26 provides

$$
f_{n}(s)=\frac{c s^{n-1}}{2 \sqrt{\pi} \Gamma(n)}(-1)^{n-1} \sqrt{\pi} \partial_{s}^{n-1}\left(s^{-1 / 2} e^{-c \sqrt{s}}\right)=\frac{s^{n-1}}{\Gamma(n)}(-1)^{n} \partial_{s}^{n}\left(e^{-c \sqrt{s}}\right) .
$$

Applying Lemma 4.1 allows to conclude.

Let us compute the expressions of the TVaR and the diversification benefit.

Proposition 4.1. Consider the dependent Weibull-Gumbel model $X=\left(X_{i}, i \geq 1\right)$ given in Theorem 4.1. Then the TVaR of the aggregate risk $S_{n}=\sum_{i=1}^{n} X_{i}$ at threshold $\kappa, 0<\kappa<1$, can be expressed, for $n \geq 1$, as:

$T V a R_{n}=\frac{2 n e^{-c \sqrt{q_{n}}}}{(1-\kappa) c^{2}}\left(1+c q_{n}^{1 / 2}+\frac{c^{2} q_{n}}{2}+\frac{c^{3} q_{n}^{3 / 2}}{4 \sqrt{\pi}} \sum_{k=2}^{n} \frac{\Gamma\left(k-\frac{3}{2}\right)}{k !}{ }_{1} F_{1}\left(2-k, 4-2 k ; 2 c \sqrt{q_{n}}\right)\right)$

with $q_{n}=F_{S_{n}}^{\leftarrow}(\kappa), F_{S_{n}}$ being defined in (24).

In particular we have, for $n=1$,

$$
T V a R_{1}=\frac{2}{c^{2}}\left(1-\ln (1-\kappa)+\frac{1}{2}(\ln (1-\kappa))^{2}\right)
$$

and, for $n=2$,

$$
T V a R_{2}=\frac{4 e^{-c \sqrt{q_{2}}}}{(1-\kappa) c^{2}}\left(1+c q_{2}^{1 / 2}+\frac{c^{2} q_{2}}{2}+\frac{c^{3} q_{2}^{3 / 2}}{8}\right)
$$

Proof. By definition of the TVaR, we can write, using the expression 27) for $f_{n}$,

$$
T V a R_{n}=\frac{1}{1-\kappa} \int_{q_{n}}^{\infty} s f_{n}(s) d s=\frac{c}{2 \sqrt{\pi}(1-\kappa) \Gamma(n)} \int_{0}^{\infty} \theta^{n-3 / 2} e^{-\frac{c^{2}}{4 \theta}} \int_{q_{n}}^{\infty} s^{n} e^{-s \theta} d s d \theta .
$$

But we have, by the change of variable $t=s \theta$,

$$
\int_{q_{n}}^{\infty} s^{n} e^{-s \theta} d s=\frac{1}{\theta^{n+1}} \Gamma\left(n+1 ; \theta q_{n}\right)=\frac{n ! e^{-\theta q_{n}}}{\theta^{n+1}} \sum_{k=0}^{n} \frac{\left(\theta q_{n}\right)^{k}}{k !},
$$

$\Gamma(s ; x)$ denoting the incomplete Gamma function defined by $\int_{x}^{\infty} t^{s-1} e^{-t} d t$ that can be expressed as a discrete sum (see [5]).

So we deduce that

$$
\begin{aligned}
\operatorname{TVaR}_{n} & =\frac{c n}{2(1-\kappa) \sqrt{\pi}}\left(\int_{0}^{\infty}\left(1+\theta q_{n}\right) \theta^{-5 / 2} e^{-\frac{c^{2}}{4 \theta}-\theta q_{n}} d \theta+\sum_{k=2}^{n} \frac{q_{n}^{k}}{k !} I_{k-2, c^{2} / 4}\left(q_{n}\right)\right) \\
& =\frac{c n}{2(1-\kappa) \sqrt{\pi}}\left(I_{1, q_{n}}\left(c^{2} / 4\right)+q_{n} I_{0, q_{n}}\left(c^{2} / 4\right)+\sum_{k=2}^{n} \frac{q_{n}^{k}}{k !} I_{k-2, c^{2} / 4}\left(q_{n}\right)\right)
\end{aligned}
$$


using the definition (26) in the first equation, and the change of variable $\theta=u^{-1}$ to compute the integral, in the second one.

On one hand, we have $I_{0, q_{n}}\left(c^{2} / 4\right)=\frac{2 \sqrt{\pi}}{c} e^{-c \sqrt{q_{n}}}$; on the other hand, a straightforward computation gives $I_{1, q_{n}}\left(c^{2} / 4\right)=\frac{4 \sqrt{\pi}}{c^{3}} e^{-c \sqrt{q_{n}}}\left(1+c \sqrt{q_{n}}\right)$. Moreover, we can write, using once again (26),

$$
\begin{aligned}
I_{k-2, c^{2} / 4}\left(q_{n}\right) & =(-1)^{k} \partial_{q_{n}}^{k-2}\left(I_{0, c^{2} / 4}\left(q_{n}\right)\right)=(-1)^{k} \sqrt{\pi} \partial_{q_{n}}^{k-2}\left(q_{n}^{-1 / 2} e^{-c \sqrt{q_{n}}}\right) \\
& =(-1)^{k-1} \frac{2 \sqrt{\pi}}{c} \partial_{q_{n}}^{k-1}\left(e^{-c \sqrt{q_{n}}}\right),
\end{aligned}
$$

from which we deduce, applying Lemma 4.1, that

$$
\sum_{k=2}^{n} \frac{q_{n}^{k}}{k !} I_{k-2, c^{2} / 4}\left(q_{n}\right)=q_{n}^{3 / 2} e^{-c \sqrt{q_{n}}} \sum_{k=2}^{n} \frac{\Gamma\left(k-\frac{3}{2}\right)}{k !}{ }_{1} F_{1}\left(2-k, 4-2 k ; 2 c \sqrt{q_{n}}\right) .
$$

Combining these results provide (28).

Applying (28) with $n=1$ provides $T V a R_{1}=\frac{2 e^{-c \sqrt{q_{1}}}}{(1-\kappa) c^{2}}\left(1+c q_{1}^{1 / 2}+\frac{c^{2} q_{1}}{2}\right)$, from which (29) follows since $q_{1}=(\ln (1-\kappa))^{2} / c^{2}$. For $n=2,30$ is a direct application of 28).

Now we can deduce the main result, namely an explicit formula of the diversification benefit associated to our model for $n$ risks.

Corollary 4.1. Consider the dependent Weibull-Gumbel model $X=\left(X_{i}, i \geq 1\right)$ given in Theorem 4.1. Then the diversification benefit of the aggregate risk $S_{n}=\sum_{i=1}^{n} X_{i}$ at threshold $\kappa$ $(0<\kappa<1)$, associated with the risk measure $\rho$, can be expressed as:

(i) For $\rho=V a R, \quad D_{n}^{*}=1-\frac{q_{n}-n \mathbb{E}(X)}{n q_{1}-n \mathbb{E}(X)}=1-\frac{c^{2}\left(q_{n}-2 n c\right)}{n\left((\ln (1-\kappa))^{2}-2 c^{3}\right)}$.

(ii) For $\rho=T V a R$,

$$
D_{n}=1-\frac{e^{-c \sqrt{q_{n}}}\left(1+c q_{n}^{1 / 2}+\frac{c^{2}}{2} q_{n}+\frac{c^{3}}{4 \sqrt{\pi}} q_{n}^{3 / 2} \sum_{k=2}^{n} \frac{\Gamma\left(k-\frac{3}{2}\right)}{k !}{ }_{1} F_{1}\left(2-k, 4-2 k ; 2 c \sqrt{q_{n}}\right)\right)-c^{3}(1-\kappa)}{(1-\kappa)\left(1-c^{3}-\ln (1-\kappa)+\frac{1}{2}(\ln (1-\kappa))^{2}\right)}
$$

which simplifies, for $n=2$, to:

$$
D_{2}=1-\frac{e^{-c \sqrt{q_{2}}}\left(1+c q_{2}^{1 / 2}+\frac{c^{2}}{2} q_{2}+\frac{c^{3}}{8} q_{2}^{3 / 2}\right)-c^{3}(1-\kappa)}{(1-\kappa)\left(1-c^{3}-\ln (1-\kappa)+\frac{1}{2}(\ln (1-\kappa))^{2}\right)}
$$

Proof.

(i) It is immediate knowing that $\mathbb{E}(X)=c \Gamma\left(1+\frac{1}{\tau}\right)=2 c$ when $X$ is $(c, \tau)$-Weibull distributed.

(ii) Combining (28), (30) respectively, with 29) in the definition of the diversification benefit (4) gives the results. 


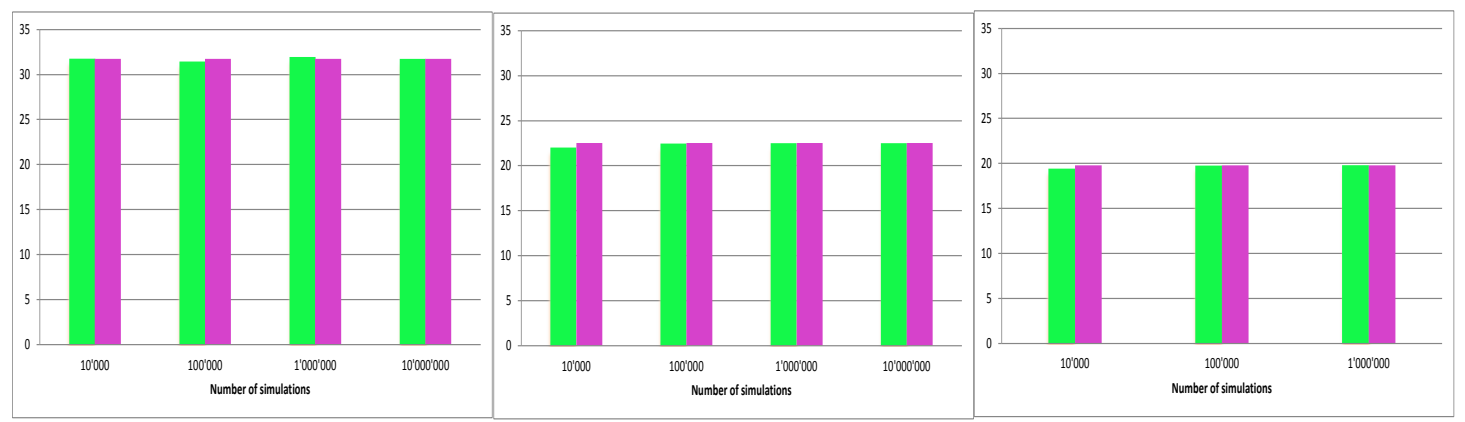

Figure 3: Convergence of the normalized TVaR of $S_{n}, T V a R_{n} / n$, at $99.5 \%$ for $c=1, \tau=\frac{1}{2}$ and $\theta=2$, for an aggregation factor $n=2,10,100$ from left to right. The purple plots are for the analytical values and the light green ones are the average values obtained from the MC simulations. The $y$-axis is the same for the three plots.

\subsection{Numerical application and discussion}

We use the analytical expressions given in Proposition 4.1 and Theorem 4.1 to compute the TVaR and the diversification benefit, for various values of the aggregation factor $n$, namely 2 , 10 and 100. Contrary to the Pareto-Clayton case, these expressions were obtained by fixing most of the parameters of the model, with the Gumbel copula parameter fixed to $\theta=2$ and the " $\tau$ " parameters of the Weibull marginals to $\tau=1 / 2$. The tail index is thus fixed to $\alpha=2$. Only the scaling parameter $c$ of the Weibull marginals might be modified, but for the sake of simplicity we fix it to 1 .

We compare these values obtained analytically with those obtained via Monte Carlo (MC) simulation, in order to check the convergence of the MC method. As for the Pareto Clayton case, we run 10 sets of simulations (changing each time the random seed of the random generator) for our set of parameters, varying the number of simulations per run from 10'000 to 10 millions (except for $n=100$, where we stop at 1 million due to computer limitations). We report here the average values of the 10 sets and verify that the standard deviation is decreasing with the number of simulations, as expected.

We present in Figure 3 the normalized TVaR, $T V a R_{n} / n$, for the 3 cases $n=2,10,100$. We see in this figure that:

- $T V a R_{n} / n$ decreases with $n$

- $T V a R_{n} / n$ decreases faster between $n=2$ to $n=10(-29 \%)$ than between $n=10$ to $n=100(-12 \%)$

- The rate of convergence is good in all the cases and the deviation reaches less than $1 \%$ already with 100'000 simulations

On the figure, the convergence is very clear. The absolute value of the relative error is less than $2 \%$ for both risk measures and for all $n$ 's. The convergence, reached already with 100'000 simulations, is faster than the one obtained with the Pareto-Clayton model. This is explained by the fact that, for $0<\tau<1$, the Weibull marginals are moderately heavy tailed and the Gumbel survival copula has a weaker dependence in the tail compared to the Clayton survival copula; thus the simulation requires less points to model accurately the behavior in the tails. Increasing the number of aggregation improves the convergence. Indeed, when measuring the variation with 1 million simulation for the TVaR, we see that for $n=2$ we have an underestimation of $0.65 \%$, which decreases to $0.18 \%$ for $n=100$. Beside the gain in precision, the analytical formula can 


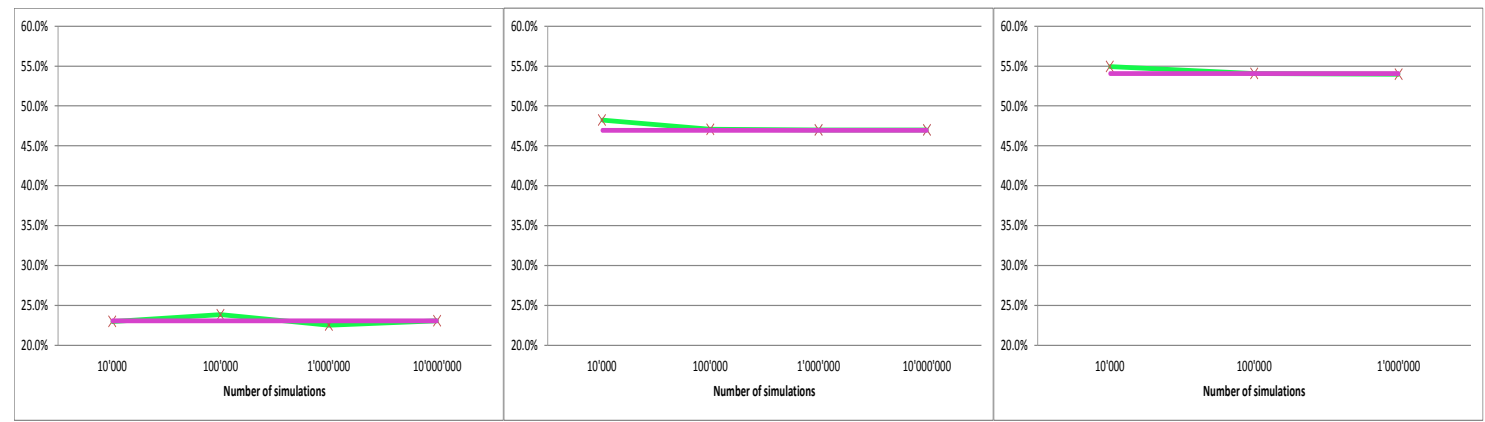

Figure 4: Convergence of the diversification benefit $D_{n}$ of $S_{n}$ (associated with TVaR at 99.5\%) for $c=1$, $\tau=\frac{1}{2}$ and $\theta=2$, for an aggregation factor $n=2,10,100$ from left to right. The purple lines are for the analytical values and the light green ones are the average values obtained from the MC simulations. The $y$-axis is the same for the three plots, for fair comparison.

be numerically evaluated 65 times faster, respectively 75 times faster (for $n=10$, resp. $n=100$ for 1 million simulation), than the estimation given by Monte Carlo (MC) method. Moreover, MC method cannot be used for higher number of aggregation (i.e $n=10^{\prime} 000$ ) due to lack of system memory, while it is of course feasible for the analytical formula.

In Figure 4, we present the results for the diversification benefit $D_{n}$, when choosing TVaR as risk measure. Similar comments hold for $D_{n}$ as for $T V a R_{n} / n$. The convergence is already very good for $10^{\prime} 000$ simulations. We also observe, as expected, that $D_{n}$ increases with $n$.

\section{Diversification benefit as a function of the aggregation factor and the risk measures}

In this section, we use the analytical expression of the diversification benefit for the two models, Pareto-Clayton (see Theorem 3.2) and Weibull-Gumbel (see Theorem 4.1) to examine and compare the behavior of the diversification benefit as a function of the aggregation factor $n$ (from $n=2$ to 10'000) and the risk measures, TVaR and VaR. For fair comparison, we choose the Pareto-Clayton case with $\alpha=2$, since it corresponds to the tail index of the Weibull-Gumbel model.

To complete the comparison, we add two cases, when having independent Pareto rv's, to evaluate the impact of the dependence, and when considering a Gaussian-Gaussian model (Gaussian marginals and Gaussian correlation) to evaluate the impact of the tail thickness. Let us recall briefly the evaluation of the diversification benefit in both cases.

Independent Pareto rv's case with asymptotic threshold ( $\kappa \nearrow 1)$

To avoid computations, we are going to look only for approximations when considering extreme quantiles (i.e. when the threshold $\kappa$ tends to 1), for which Feller's result (see [4]) is available. For sharper and not necessarily asymptotic results, computations could be done using the Normex method (see [8]).

Feller has shown that the tail distribution of the sum of independent rv's with regularly varying $R V_{\alpha}$ tail distribution is asymptotically $R V_{\alpha}$. Applying this result when considering iid Pareto- 
$(\alpha, \beta)$ rv's provides

$$
q_{n}=\operatorname{VaR}_{\kappa}\left(S_{n}\right)=F_{S_{n}}^{\leftarrow}(\kappa) \underset{\kappa \rightarrow 1}{\sim} \beta\left(\left(\frac{1-\kappa}{n}\right)^{-1 / \alpha}-1\right)
$$

and

$$
\operatorname{TVaR}_{\kappa}\left(S_{n}\right) \underset{\kappa \rightarrow 1}{\sim} \frac{n \beta^{\alpha}}{(1-\kappa)(\alpha-1)} \times \frac{\alpha q_{n}+\beta}{\left(q_{n}+\beta\right)^{\alpha}} \underset{\kappa \rightarrow 1}{\sim} \beta\left(\frac{\alpha}{\alpha-1}\left(\frac{1-\kappa}{n}\right)^{-1 / \alpha}-1\right) .
$$

Note that we get back the asymptotic relation $T V a R_{\kappa} / V a R_{\kappa} \rightarrow \alpha /(\alpha-1)$ as $\kappa \rightarrow 1$.

Now let us look at the diversification benefit for high threshold $\kappa$. When choosing the VaR as risk measure ( $q_{1}$ satisfying (6p), we have

$$
D_{n}^{*}(\kappa)=1-\frac{q_{n}-\frac{n \beta}{\alpha-1}}{n\left(q_{1}-\frac{\beta}{\alpha-1}\right)} \underset{\kappa \rightarrow 1}{\sim} 1-\frac{n^{\frac{1}{\alpha}-1}(1-\kappa)^{-1 / \alpha}-\frac{1}{n}-\frac{1}{\alpha-1}}{(1-\kappa)^{-1 / \alpha}-1-\frac{1}{\alpha-1}}
$$

and for the TVaR,

$$
D_{n}(\kappa) \underset{\kappa \rightarrow 1}{\sim} 1-\frac{\frac{\alpha}{\alpha-1} n^{\frac{1}{\alpha}-1}(1-\kappa)^{-1 / \alpha}-\frac{1}{n}-\frac{1}{\alpha-1}}{\frac{\alpha}{\alpha-1}(1-\kappa)^{-1 / \alpha}-1-\frac{1}{\alpha-1}}
$$

from which we deduce the asymptotic limit as $\kappa \rightarrow 1$, which is the same for both risk measures, as expected:

$$
\lim _{\kappa \rightarrow 1} D_{n}^{*}(\kappa)=\lim _{\kappa \rightarrow 1} D_{n}(\kappa)=1-n^{-(1-1 / \alpha)}
$$

which tends to 1 as $n \rightarrow \infty$.

Note that in the Gaussian case, $D_{n}$ (or $D_{n}^{*}$ ), converges also to 1 as $n \rightarrow \infty$ with a rate of convergence of $n^{1 / 2}$, and not only for high threshold $\kappa$.

\section{Multivariate Gaussian distribution}

Consider the Gaussian vector $\left(X_{i}\right)_{i=1, \cdots, n}$ with expectation vector $\left(\mu_{i}\right)_{i=1, \cdots, n}, \mu_{i}=\mu$, and (non negative definite) covariance matrix $\Gamma=\left(\gamma_{i j}\right)_{1 \leq i, j \leq n}$ such that $\gamma_{i i}=\sigma^{2}, \forall i$. Then $S_{n}=\sum_{i=1}^{n} X_{i}$ is normally distributed with mean $n \mu$ and variance $n \sigma^{2}+2 \sum_{1 \leq i<j \leq n} \gamma_{i j}$. Hence, with the notation $r_{i j}=\operatorname{corr}\left(X_{i}, X_{j}\right)$ and $\phi, \Phi$, for the standard normal probability density function, cumulative distribution function respectively, we can write

$$
q_{n}=\operatorname{VaR}_{\kappa}\left(S_{n}\right)=n \mu+\Phi^{-1}(\kappa) \sqrt{n} \sigma \sqrt{1+\frac{2}{n} \sum_{1 \leq i<j \leq n} r_{i j}} \leq n\left(\mu+\Phi^{-1}(\kappa) \sigma\right)=n q_{1},
$$

whereas, for the TVaR,

$T \operatorname{Ta} R_{\kappa}\left(S_{n}\right)=n \mu+\frac{\phi\left(\Phi^{-1}(\kappa)\right)}{1-\kappa} \sqrt{n} \sigma \sqrt{1+\frac{2}{n} \sum_{1 \leq i<j \leq n} r_{i j}} \leq n\left(\mu+\frac{\phi\left(\Phi^{-1}(\kappa)\right)}{1-\kappa} \sigma\right)=n T \operatorname{Ta} R_{\kappa}(X)$.

We deduce that

$$
D_{n}=D_{n}^{*}=1-\frac{1}{\sqrt{n}} \sqrt{1+\frac{2}{n} \sum_{1 \leq i<j \leq n} r_{i j}}(\geq 0)
$$


Table 2: Analytical diversification benefit of $S_{n}, D_{n}$ and $D_{n}^{*}$, at $99.5 \%$ as a function of the risk measures $\mathrm{TVaR}$ and $\mathrm{VaR}$, respectively, and of the aggregation factor $n$. Tail index $\alpha=2$, survival Clayton parameter $\theta=1 / 2$, Weibull $\tau=1 / 2$, Gumbel parameter $\theta=2$.

\begin{tabular}{|c|c|c|c|c|c|}
\hline & $\mathrm{n}=2$ & $\mathrm{n}=10$ & $\mathrm{n}=100$ & $\mathrm{n}=1000$ & $\mathrm{n}=10^{\prime} 000$ \\
\hline \multirow{2}{*}{\multicolumn{6}{|c|}{$\begin{array}{c}\text { Independent Pareto } \\
D_{n}=D_{n}^{*}\end{array}$}} \\
\hline & $29.3 \%$ & $68.4 \%$ & $90.0 \%$ & $96.8 \%$ & $99.0 \%$ \\
\hline \multicolumn{6}{|l|}{ Pareto-Clayton } \\
\hline$D_{n}$ & $13.2 \%$ & $25.5 \%$ & $28.6 \%$ & $29.0 \%$ & $29.0 \%$ \\
\hline$D_{n}^{*}$ & $12.9 \%$ & $25.2 \%$ & $28.3 \%$ & $28.6 \%$ & $28.7 \%$ \\
\hline$D_{n} / D_{n}^{*}$ & 1.021 & 1.014 & 1.012 & 1.012 & 1.012 \\
\hline \multicolumn{6}{|l|}{$\begin{array}{l}\text { Gaussian-Gaussian } \\
r=0.42 \text { (Clayton) }\end{array}$} \\
\hline$D_{n}=D_{n}^{*}$ & $15.7 \%$ & $30.9 \%$ & $34.7 \%$ & $35.1 \%$ & $35.2 \%$ \\
\hline \multicolumn{6}{|l|}{ Weibull-Gumbel } \\
\hline$D_{n}$ & $23.1 \%$ & $47.0 \%$ & $54.1 \%$ & $54.8 \%$ & $54.9 \%$ \\
\hline$D_{n}^{*}$ & $19.6 \%$ & $40.4 \%$ & $46.5 \%$ & $47.2 \%$ & $47.2 \%$ \\
\hline$D_{n} / D_{n}^{*}$ & 1.179 & 1.162 & 1.163 & 1.163 & 1.163 \\
\hline \multicolumn{6}{|l|}{$\begin{array}{l}\text { Gaussian-Gaussian } \\
r=0.39 \text { (Gumbel) }\end{array}$} \\
\hline$D_{n}=D_{n}^{*}$ & $16.6 \%$ & $32.8 \%$ & $37.1 \%$ & $37.5 \%$ & $37.5 \%$ \\
\hline
\end{tabular}

The diversification benefit can tend to any constant between 0 and 1 , as $n \rightarrow \infty$, whenever there exists a linear dependence between the components. For instance, if $r_{i j}=r \neq 0, \forall i \neq j$, the diversification benefit reduces to

$$
D_{n}=D_{n}^{*}=1-\frac{1}{\sqrt{n}} \sqrt{1+(n-1) r} .
$$

When $r=1$ (full comonotonicity), $D_{n}=D_{n}^{*}=0$, whereas for $r=0$ (independent case), we get back that $D_{n}=D_{n}^{*}=1-n^{-1 / 2}$, which is also the limit (as $\left.\kappa \rightarrow 1\right)$ of the diversification benefit given in (37) for independent $\alpha$-Pareto rv's with $\alpha=2$.

Let us compare the diversification benefit as a function of $n$, for both TVAR and VaR, for high threshold $\kappa=99.5 \%$, considering the following cases: (i) independent $\alpha$-Pareto with $\alpha=2$, (ii) $\alpha$-Pareto margins and survival Clayton $(\theta)$ copula (Pareto-Clayton) with $\alpha=2=1 / \theta$, (iii) Gaussian margins and Gaussian copula (Gaussian-Gaussian) with (linear) correlation $r=0.42$ estimated on the previous Pareto-Clayton model, (iv) $\tau$-Weibull margins and Gumbel $(\theta)$ copula (Weibull-Gumbel) with $\theta=2=1 / \tau$, and (v) Gaussian-Gaussian with correlation $r=0.39$ estimated on the Weibull-Gumbel model.

The linear correlation coefficients are estimated from the realization of the simulated model. We choose the number of simulations for which the Kendall $-\tau$ estimate corresponds to the 


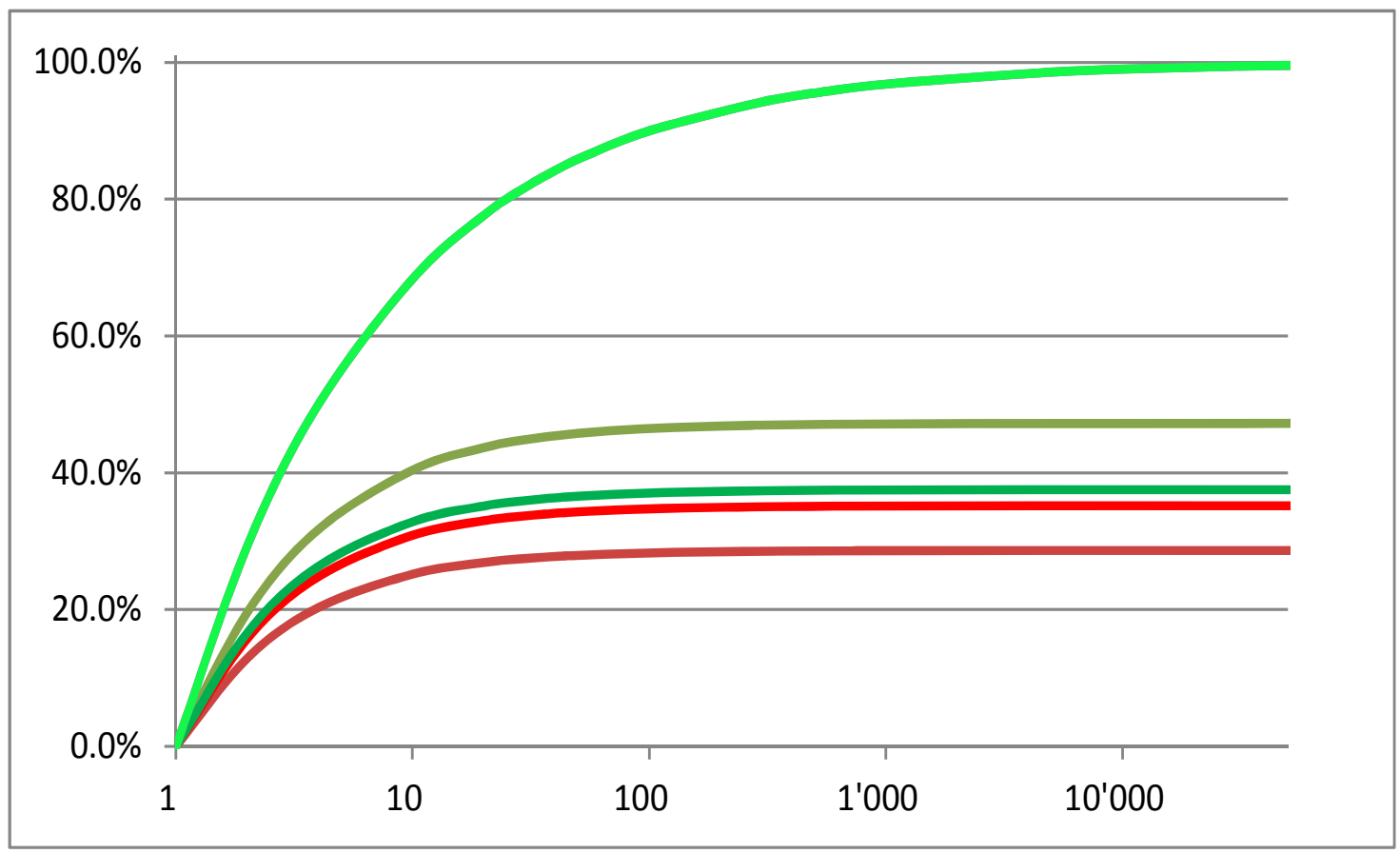

Figure 5: Plot of the diversification benefit $D_{n}^{*}$ (y-axis) as a function of $n$ ( $x$-axis), for $\operatorname{VaR} R_{\kappa}$ with $\kappa=99.5 \%$. The curves from bottom to up correspond, respectively, to Pareto $(\alpha=2)$-Clayton $(\theta=1 / 2)$, Gauss-Gauss (Clayton; $r=0.42)$, Gauss-Gauss (Gumbel; $r=0.39)$, Weibulll $(\tau=1 / 2)$-Gumbel $(\theta=2)$, independent Pareto and Gauss models $(\alpha=2)$.

theoretical one. Recall that the theoretical value of $\tau$, as a function of the $\theta$ parameter of the copula, is known for both Clayton, with $\tau=\frac{\theta}{\theta+2}$, and Gumbel, with $1-\frac{1}{\theta}$. The convergence is reached with 100'000 simulations.

In Table 2, we present the results obtained on $D_{n}$ and $D_{n}^{*}$ using analytical formulas for all of them, and not simulations. Note that it completes the numerical application for the analytical diversification benefit done in the previous sections with the TVaR only. Moreover, the functions $D_{n}^{*}$ of $n$ are plotted in Figure 5 for the various models.

We show in Table 2 and Figure 5 that:

- The dependence has much impact on the evolution of the diversification benefit with $n$, as expected. In the case of dependence, the diversification benefit levels off rapidely, already at $n=100$, while for the independence case, it still increases from $n=100$ to $n=10^{\prime} 000$

- The evolution of the diversification benefit is similar for the two underlying structures, increasing and leveling off with $n$, for both. For example, in the dependent case, from $n=2$ to 10 , it increases approximately by a factor 2 (1.9 for Pareto-Clayton and 2.0 for Weibull-Gumbel), regardless of the risk measure used to compute the diversification benefit

- Although both evolutions are the same from $n=2$ to 100 , the Pareto-Clayton model exhibits a stronger tail dependence and a fatter tail than the Weibull-Gumbel one. This appears through the difference of the diversification benefit between the two models, almost twice bigger for the second

- We see in the table that the diversification benefit computed with the VaR is always 
lower than the one computed with the TVaR. This is to be expected, since the latter risk measure takes into account the whole distribution beyond a fixed threshold (for instance 99.5\% in this case), whereas the VaR only looks at one point of the distribution. However, the difference is more pronounced for the Weibull-Gumbel model than the Pareto-Clayton one, with a ratio of the order of 1.2 instead of 1.01 respectively

- The ratio $D_{n} / D_{n}^{*}$ stabilizes from $n=10$ onwards, with 1.012 for Pareto-Clayton and 1.163 for Weibull-Gumbel. Starting at $n=100$, the diversification benefit does not change anymore

- Comparing the results obtained with the Pareto-Clayton model and the corresponding Gaussian-Gaussian one, emphasizes that the tail dependence associated with a fat tail limits the diversification benefit. The diversification benefit limit is $35.2 \%$ for the Gaussian case, while it is only $29 \%$ in the Pareto-Clayton case

- The comparison leads to a different result in the Weibull-Gumbel case and the Gaussian one. Here we see much stronger diversification benefit for the Weibull-Gumbel model than for the Gaussian-Gaussian one. The intuition to explain this observation being less obvious than with the previous model, other examples varying the parameters would help to analyze this effect. Let us just note that the linear correlation implied by $\theta=2$ is quite high in this case

- As expected, $100 \%$ diversification benefit is reached in the independent case only.

\section{Conclusion}

In this paper, we use the mixing techniques to compute explicitly the probability density function (pdf) of n-aggregated risks for two combinations of dependence structure and marginal distributions, Pareto-Clayton and Weibull-Gumbel. From this pdf, we have derived the explicit formulas for the TVaR, the VaR and the diversification benefit. These formulas are then used to study the convergence of the TVaR and the diversification benefit as a function of the tail indices. By varying the number of simulations from 10'000 to 10 millions, we find out that for Pareto-Clayton a minimum of 100'000 simulations is needed to obtain a good convergence for moderately heavy tails $\alpha=2$ to $\alpha=3$. For very heavy tails, as e.g. $\alpha=1.1$, we do not reach convergence, even with 10 millions simulations, showing that we must be careful when using MC method for this kind of distributions. The second case is more limited since the Gumbel copula parameter is fixed to $\theta=2$, however we can conclude that the convergence with a Gumbel copula is faster than with a Clayton survival copula since 100'000 simulations are considered as sufficient for moderate heavy tail Weibull distribution.

In addition, we study the behavior of the analytical diversification benefit of $S_{n}$ as a function of the aggregation factor $n$. The variation is similar for the two structures. For example, it increases approximately by a factor 2 from $n=2$ to $n=100$. The diversification benefit for large $\mathrm{n}$ converges to a lower value for the Pareto-Clayton model than for the Gumbel-Weibull one, due to the fact that the tails and the dependence in the tail are stronger for the first model. When comparing it to the one obatined with an elliptical model (Gaussian copula), we observe two opposite behaviors depending on the considered model. In the Pareto-Clayton case, the diversification benefit of the Gaussian model converges clearly to a higher value, while we see the reverse for the Gumbel-Weibull case. It is also interesting to note that in the independent case, Pareto with $\alpha=2$ has an identical behavior as the Gaussian one, despite the fact that 
Pareto has a heavier tail. Moreover, as expected, the diversification benefit computed with the VaR is lower than the one computed with the TVaR, however this difference stabilizes when increasing the aggregation factor $n$.

Bypassing Monte Carlo simulation is a considerable gain in precision and time. Indeed, the analytical formulas are estimated to be 40 to 500 times faster than $\mathrm{MC}$ method with the difference increasing with increasing n's. Moreover, with the analytical formula we can estimate the TVaR even for very heavy tail distributions when MC method fails even for 10 millions simulations. Explicit formulas let us also explore the aggregation behavior of the risk measures and the diversification benefit. It is a precious tool for validating results of internal models, which are based on MC simulations. Numerical methods should be used only when no anayltical solutions are available.

Acknowledgement: Partial support from RARE-318984 (an FP7 Marie Curie IRSES Fellowship) is kindly acknowledged.

\section{References}

[1] M. Albano, T. Amdeberhan, E. Beyerstedt, V.H. Moll (2011), The integrals in Gradshteyn and Ryzhik. Part 19 : The error function, SCIENTIA Series A: Mathematical Sciences 21, 25-42

[2] H. Albrecher, C. Constantinescu, S. Loisel (2011), Explicit ruin formulas for models with dependence among risks, Insurance: Mathematics and Economics 48(2) 265-270

[3] R. Bürgi, M.M. Dacorogna, R. Iles (2008), Risk Aggregation, dependence structure and diversification benefit. In: Stress testing for financial institutions, Riskbooks, Incisive Media, London, 265-306.

[4] W. Feller (1966), An introduction to probability theory and its applications, Vol.II. Wiley.

[5] I.S. Gradshteyn (1988), Tables of Integrals, Series, and Products.

[6] P. A. GroenendiJk, A. Luchs, C. DE VRIEs (1996), Stochastic processes, non-normal innovations, and the use of scaling ratios, In Proceedings of the third International Conference on Forecasting Financial Markets, London, 1-38.

[7] K. T. Kohl and V.H. Moll (2011), The integrals in Gradshteyn and Ryzhik . Part 20 : Hypergeometric functions, SCIENTIA Series A: Mathematical Sciences 21, 43-54.

[8] M. Kratz (2014), Normex, a new method for evaluating the distribution of aggregated heavy tailed risks. Application to risk measures. Extremes 17(4), Special issue on Extremes and Finance (Guest Ed. P. Embrechts), 661-691

[9] A.W. Marshall and I. Olkin (1988), Families of Multivariate Distributions, Journal of the American Statistical Association 83, 834-841.

[10] D. OAkes (1989), Bivariate Survival Models Induced by Frailties, Journal of the American Statistical Association 84, 487-493. 


\section{PARIS}

ESSEC Business School

3, avenue Bernard-Hirsch

CS 50105 Cergy

95021 Cergy-Pontoise Cedex

France

Tél. + 33 (0) 134433000

www.essec.fr
ESSEC Executive Education

CNIT BP 230

92053 Paris-La Défense

France

Tél. + 33 (0)146924900

www.executive-education.essec.fr
ESSEC Asia Pacific

2 One-North Gateway

Singapore 138502

Tél. +65 68849780

www.essec.edu/asia

\section{- SINGAPOUR}

\section{Contact :}

Centre de Recherche

+33 (0)134433091

research.center@essec.fr 